\title{
Appropriate Clinical Strategies for Breast Cancer Coexisting with Acute Myeloid Leukemia in the Genomic-Molecular Era: A Case Report
}

\author{
Guangfu Hu ${ }^{a}$ Dipendra K. Mallik ${ }^{a}$ Weige Yang ${ }^{a}$ Yingyong Hou ${ }^{b}$ Zhixiang Cheng ${ }^{\mathrm{c}}$ \\ Pu Chen ${ }^{c}$ Wei Zhu $^{a}$ Hong Wang $^{a}$ Lei Shen ${ }^{a}$ Hongwei Zhang ${ }^{\text {a }}$ Ziang Yang $^{\mathrm{a}}$ \\ ${ }^{a}$ Department of General Surgery, Zhongshan Hospital, Fudan University, Shanghai, China; \\ ${ }^{b}$ Department of Pathology, Zhongshan Hospital, Fudan University, Shanghai, China; \\ ${ }^{\mathrm{c}}$ Hematological Department, Zhongshan Hospital, Fudan University, Shanghai, China
}

\section{Established Facts}

- The management of a synchronous occurrence of breast cancerand acute myeloid leukemia is based on treating the acute myeloid leukemia immediately while putting breast cancer treatment on hold.

\section{Novel Insights}

- For a certain group of patients whose prognosis of acute myeloid leukemia is good, simultaneous or initial treatment of breast cancer before treatment of acute myeloid leukemia may be appropriate.

\section{Keywords}

Breast cancer - Acute myeloid leukemia

\section{Summary}

Background: The coexistence of breast cancer (BC) and acute myeloid leukemia (AML) has rarely been reported. Considering the fatality of $A M L$, the management of this condition is based on treating the AML immediately while putting BC treatment on hold. Case Report: Here, we report a synchronous occurrence of $B C$ and $A M L$. Prognostic factors for both $B C$ and $A M L$ were determined by genomic and molecular evaluation. The evaluation for AML showed a relatively good prognosis, and we simultaneously conducted treatment for $\mathrm{AML}$ and $\mathrm{BC}$. The patient has survived for more than 3 years, which makes this the case with the longest survival reported. Conclusion: In patients with $B C$ and $A M L$, it is essential to deter-

Guangfu Hu and Dipendra Kumar Mallik contributed equally to this work. mine the prognosis through a genomic and molecular evaluation. For a certain group of patients whose prognosis of AML is good, simultaneous or initial treatment of $\mathrm{BC}$ before treatment of AML may be appropriate.

(c) 2016 S. Karger GmbH, Freiburg

\section{Introduction}

The synchronous occurrence (within 6 months) of breast cancer (BC) and acute myeloid leukemia (AML) has rarely been reported in the past [1]. To this day, clinicians treat the AML first before treating the $\mathrm{BC}$. In the genomic-molecular era, through the evaluation of the FLT3/ITD, C-KIT, NPM1, and CEBPA gene mutation status, the prognosis of AML can be determined [2]. Thus, screening for patients with a good prognosis is now possible. Here, we present the case of a patient whose genomic and molecular evaluation for AML showed a relatively good prognosis. Based on this, it

\section{KARGER \\ Fax +497614520714

\section{(c) 2016 S. Karger GmbH, Freiburg}

$1661-3791 / 16 / 0112-0145 \$ 39.50 / 0$ 
Fig. 1. Hematoxylineosin staining showing grade III invasive ductal carcinoma $(\times 100)$.

Fig. 2. Cellular bone marrow $\mathrm{G} / \mathrm{E}=2.64$, with $76 \%$ blasts $(\times 100)$.
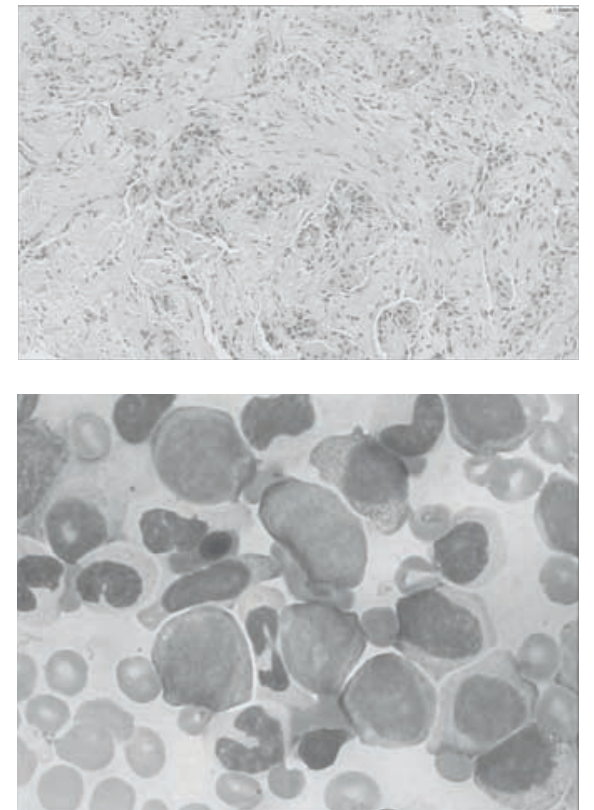

was decided to conduct AML and BC treatment simultaneously. Despite bone metastases, the patient's quality of life remarkably improved after modified radical mastectomy (MRM), suggesting the necessity of active intervention in this case.

\section{Case Report}

A 57-year-old premenopausal female patient was admitted to our hospital in October 2012 because of 2 right-sided breast masses with skin ulceration. Ultrasound showed that 1 mass was $21 \times 22 \mathrm{~mm}$ in size ( 7 o'clock) while the other measured $8 \times 9 \mathrm{~mm}$ (9 o'clock) with ipsilateral axillary lymphadenopathy. An ultrasound-guided core needle biopsy confirmed grade III invasive ductal carcinoma (fig. 1). Immunohistochemistry (IHC) for estrogen, progesterone, and human epidermal growth factor receptor 2 (HER2) was strongly positive, together with a high Ki-67 proliferation index ( $20 \%+)$; however, fluorescent in situ hybridization was negative, indicating luminal B-like (HER2-negative) type BC (staging T4N1M0). The patient was recommended to receive neoadjuvant chemotherapy before MRM following the assessment of tumor sensitivity to chemotherapy.

Incidentally, a complete blood count revealed a low hemoglobin level (9.6 g/ dl) and significant increase in total leucocytes $\left(18.5 \times 10^{9} / 1\right)$; a differential count revealed neutrophils $22 \%$, lymphocytes $15 \%$, abnormal cells $60 \%$, and platelets $117 \times 10^{9} / 1$. Following the suspicion of leukemia, further examinations revealed cellular bone marrow ( $\mathrm{G} / \mathrm{E}=2.64$, with $76 \%$ blasts) (fig. 2). Blood slide classification showed $53 \%$ of blasts; IHC verified that these blasts were negative for periodic acid-Schiff stain, napthyl acetate esterase (NAE) stain, and NAE + sodium fluoride stain, and positive for peroxidase stain. Immuno-phenotypic characteristics of the bone marrow showed that the blasts were positive for human leucocyte antigen-DR (HLA-DR), cluster of differentiation (CD) 117, CD34, CD33, CD64, CD11c, CD13, CD38, and cMPO. Molecular and cytogenetic analysis detected an abnormal karyotype: $47, \mathrm{XX},+11(100 \%)$ (fig. 3). An evaluation of the gene mutation status and leukemia fusion gene analysis demonstrated negativity for FLT3/ITD, C-KIT, and all 31-fusion genes, and positivity for NPM1 and CEBPA. Therefore, this case was classified as mature M4, type IV AML.

AML treatment began with standard daunorubicin $60 \mathrm{mg}$ days 1-3 and arabinoside cytarabine $200 \mathrm{mg}$ days 1-7 induction chemotherapy on 2 occasions in November 2012 and in January 2013. However, both attempts failed with marrow blast occupancy of 41.5 and $16 \%$, respectively. A third round of chemotherapy with CAG (arabinoside cytarabine $25 \mathrm{mg}$ days 1-14, aclarubicin $10 \mathrm{mg}$ days $1-8$, and granulocyte-colony stimulating factor (G-CSF) $300 \mu \mathrm{g}$ days 1-14) was

\section{ge

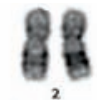 \\ 8 \\ !. \\ (3)}

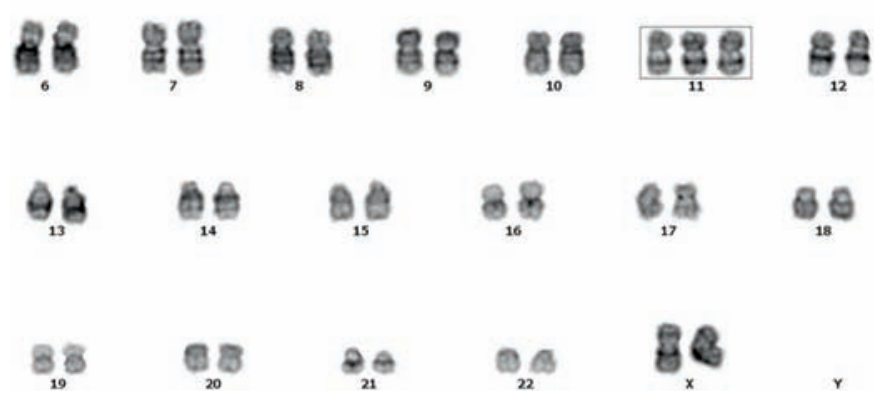

Fig. 3. Molecular and cytogenetic analysis showing an abnormal karyotype: $47, \mathrm{XX},+11(100 \%)$.
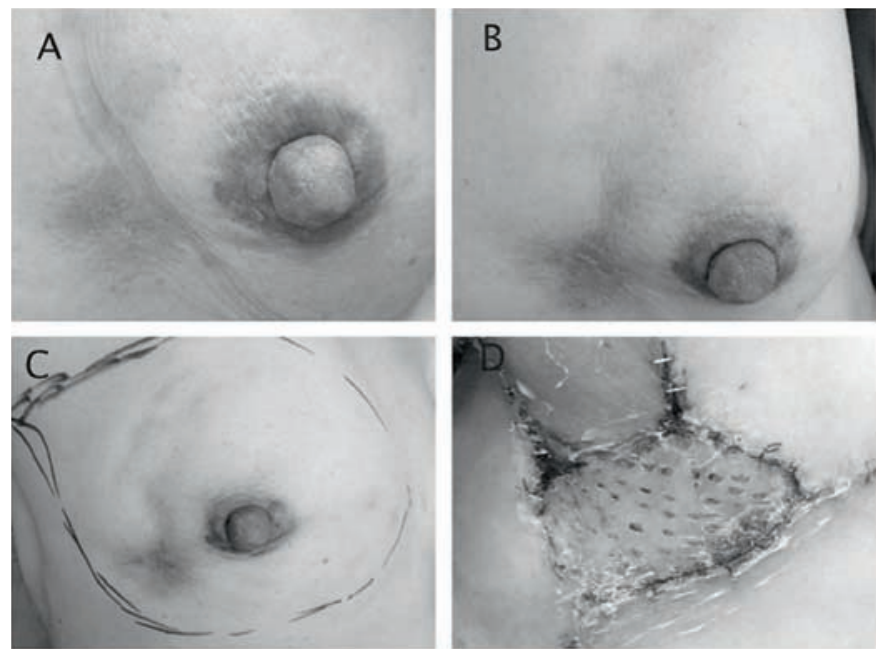

Fig. 4. The patient underwent modified radical mastectomy (MRM) and an autograft of the inguinal region. A Breast cancer (BC) progression after chemotherapy for acute myeloid leukemia; B Post 3rd chemotherapy session for BC; C Post 5th chemotherapy session for BC; D 3rd week post surgery.

carried out in March 2013 and led to complete remission (CR) with marrow blast occupancy of $2 \%$. Subsequently, the patient received arabinoside cytarabine (4.0 g days 1-3) 6 times from April 2013 to December 2013. As she suffered from level IV bone marrow inhibition accompanied by fever, vancomycin and meropenem were administered, together with thrombopoietin and G-CSF treatment. During surveillance, the patient's condition was stable without relapse.

Meanwhile, tamoxifen was prescribed for BC from October 2012; however, treatment was terminated 2 weeks later due to high fever, irritation, and insomnia. From January 2013 to November 2013, capecitabine ( $1.5 \mathrm{~g}$ twice daily) was administered for 7 cycles within 3 weeks. Until December 2013, the patient achieved CR for leukemia; however, the right-sided BC progressed from 2 to 3 lesions, and satellite nodules relapsed and even metastasized to the head of the humerus. Next, from December 2013, TEC therapy (paclitaxel $77 \mathrm{mg} / \mathrm{m}^{2}$, epirubicin $77 \mathrm{mg} / \mathrm{m}^{2}$, cyclophosphamide $0.6 \mathrm{~g} / \mathrm{m}^{2}$, on day 1 , every 3 weeks, 6 cycles) was conducted for BC. By May 2014, after 6 cycles of TEC therapy, BC borders had shrunk and satellite nodules had decreased.

In summary, the patient achieved CR for leukemia while her BC initially progressed but then became stable. To improve clinical outcome, in May 2014, she underwent MRM with an autograft from the inguinal region (fig. 4 A-D). Post- and pre-operative IHC results were matched. From May 2014 up to January 2016, the patient has been maintained with letrozole ( $2.5 \mathrm{mg}$ daily) and zoledronic acid (4 mg monthly) with the follow-up review showing her condi- 
tion to be stable. The bone metastatic lesion showed no progression on radionuclide imaging, and no signs of recurrence of AML have been observed in bone marrow and blood smear examinations.

The study protocol was approved by the ethics review board of Zhongshan Hospital. Written informed consent was obtained from the patient. All procedures were in accordance with the Declaration of Helsinki and relevant policies in China.

\section{Discussion}

Including our case, only 7 cases of such a synchronous occurrence of BC and AML have been reported since 1965 [1]. In these 7 patients, 4 survived for as little as 1 week to 1.5 months, and only 1 patient lived for another 1.5 years. All patients died of AML. However, our patient has survived for more than 3 years which makes this the case with the longest survival of all the reported cases. Besides, our report is the first description, to our knowledge, of a genomic and molecular evaluation of prognostic factors for both $\mathrm{BC}$ and AML. Our case indicates that the consideration of specific AML gene mutations and $\mathrm{BC}$ molecular subtypes is crucial when deciding on a treatment strategy for this extremely rare entity.

There are certain genetic and chromosomal events related to the co-occurrence of $\mathrm{BC}$ and AML. The p53 mutation is present as an acquired defect among $20-40 \%$ of BC patients and $10-20 \%$ of AML patients $[3,4]$. The presence of a p53 mutation is associated with a dismal prognosis. Alternative mechanisms involving p53 in AML with $\mathrm{t}(8 ; 21)$ chromosomal abnormality for creating AML1ETO fusions enable the cells to bypass the regulatory effect of p53 [5]. In our patient, the 31-fusion genes including the AML1-ETO fusion gene were all negative, which means a p53 mutation-negative status and good prognosis.

Notably, chromosome 11 features in the cytogenetic analysis of both BC and AML [6, 7]. In AML, chromosome 11 is one of the most common targets of gene amplification [8]. Amplicons on chromosome 11 may vary in size, but they usually involve the long arm of the chromosome, particularly the region 11q23, where an MLL proto-oncogene (myeloid/lymphoid leukemia or mixed-lineage leukemia (trithorax homolog, Drosophila)) is located [9]. In $\mathrm{BC}$, nonrandom abnormalities of chromosome 11 are frequently reported, with involvement of $11 \mathrm{p} 15,11 \mathrm{q} 13$, and $11 \mathrm{q} 23$ [6]. Trisomy 11 is associated with unfavorable prognosis in AML but not with NPM1 or KIT mutation [10]. Chromosomal imbalances are associated with metastasis-free survival duration in BC patients, and gain of chromosome 11 in $\mathrm{BC}$ is an indicator of a favorable prognosis [11]. Similarly, our patient had cytogenetic abnormalities of trisomy $11(47, \mathrm{XX},+11(100 \%))$, which indicates a good prognosis for $\mathrm{BC}$ but a poor prognosis for AML.

In 2010, an international expert panel (European Leukemia Net, ELN) established a new classification for AML based on molecular and cytogenetic markers [2]; the evaluation of the FLT3/ITD, C-KIT, NPM1, and CEBPA gene mutation status can determine the prognosis of AML. Meanwhile, molecular subtypes of $\mathrm{BC}$ cover luminal A-like, luminal B-like, HER2-positive, and triple-negative, referring to the St. Gallen consensus [12]. Our patient demonstrated luminal B-like (HER2-negative) type BC and was NPM1-, CEBPA-positive and FLT3/ITD-, C-KIT-negative for AML, all of which indicate a relatively good prognosis for $\mathrm{BC}$ and $\mathrm{AML}$.

All in all, the factors determining the prognosis of $\mathrm{BC}$ and $\mathrm{AML}$ were relatively good in our patient. Hence, it was decided to conduct endocrine therapy or oral chemotherapy for $\mathrm{BC}$ within the same time interval as chemotherapy for AML, followed by MRM for BC. Had this patient been admitted earlier, hence allowing for breastconserving surgery before the progression of malignancy, we believe that her prognosis may have been much more favorable. The MRM procedure was carried out in the presence of bone metastases, and yet the patient's quality of life improved remarkably after surgery.

In conclusion, it is essential to determine the prognosis and select appropriate treatment measures in patients with coexisting $\mathrm{BC}$ and AML in the genomic-molecular era. For a certain group of patients whose prognosis of AML is good, simultaneous or initial treatment of $\mathrm{BC}$ before treatment of AML may be appropriate. Further studies are required to clarify the potential biological behaviors of such cases and optimize the therapeutic strategy.

\section{Disclosure Statement}

The authors have no conflicts of interest.

\section{References}

1 Mishra PP, Mahapatra M, Choudhry VP, et al.: Synchronous occurrence of breast carcinoma and acute myeloid leukemia: case report and review of the literature. Ann Hematol 2004;83:541-543.

2 Dohner H, Estey EH, Amadori S, et al.: Diagnosis and management of acute myeloid leukemia in adults: recommendations from an international expert panel, on behalf of the European Leukemia Net. Blood 2010;115: 453-474.

3 Shiovitz S, Korde LA: Genetics of breast cancer: a topic in evolution. Ann Oncol 2015;26:1291-1299.

4 Bally C, Ades L, Renneville A, et al.: Prognostic value of TP53 gene mutations in myelodysplastic syndromes and acute myeloid leukemia treated with azacitidine. Leuk Res 2014;38:751-755.
Linggi B, Müller-Tidow C, van de Locht L, et al.: The $\mathrm{t}(8 ; 21)$ fusion protein, AML1 ETO, specifically represses the transcription of the p14ARF tumor suppressor in acute myeloid leukemia. Nat Med 2002;8:743-750.

6 Sarova I, Brezinova J, Zemanova Z, et al.: Characterization of chromosome 11 breakpoints and the areas of deletion and amplification in patients with newly diagnosed acute myeloid leukemia. Genes Chromosomes Cancer 2013;52:619-635.

7 Tan PH, Lui WO, Ong P, et al.: Cytogenetic analysis of invasive breast cancer: a study of 27 Asian patients. Cancer Genet Cytogenet 2000;121:61-66.

8 Zatkova A, Merk S, Wendehack M, et al.: AML/MDS with 11q/MLL amplification show characteristic gene expression signature and interplay of DNA copy number changes. Genes Chromosomes Cancer 2009;48:510-520.
Klampfl T, Milosevic JD, Puda A, et al.: Complex patterns of chromosome 11 aberrations in myeloid malignancies target CBL, MLL, DDB1 and LMO2. PloS One 2013;8:e77819.

10 Alseraye FM, Zuo Z, Bueso-Ramos C, et al.: Trisomy 11 as an isolated abnormality in acute myeloid leukemia is associated with unfavorable prognosis but not with an NPM1 or KIT mutation. Int J Clin Exp Pathol 2011;4:371.

11 Aubele M, Auer G, Braselmann H, et al.: Chromosomal imbalances are associated with metastasis-free survival in breast cancer patients. Anal Cell Pathol 2002;24:77-87.

12 Goldhirsch A, Wood WC, Coates AS, et al.: Strategies for subtypes - dealing with the diversity of breast cancer: highlights of the St. Gallen International Expert Consensus on the Primary Therapy of Early Breast Cancer 2011. Ann Oncol 2011;22:1736-1747. 